\title{
HIF3A association with adiposity: the story begins before birth
}

\begin{abstract}
Aim: Determine if the association of HIF3A DNA methylation with weight and adiposity is detectable early in life. Material \& methods: We determined HIF3A genotype and DNA methylation patterns (on hybridization arrays) in DNA extracted from umbilical cords of 991 infants. Methylation levels at three CpGs in the HIF3A first intron were related to neonatal and infant anthropometry and to genotype at nearby polymorphic sites. Results \& conclusion: Higher methylation levels at three previously described HIF3A CpGs were associated with greater infant weight and adiposity. The effect sizes were slightly smaller than those reported for adult BMI. There was also an interaction within cis-genotype. The association between higher DNA methylation at HIF3A and increased adiposity is present in neonates. In this study, no particular prenatal factor strongly influenced HIF3A hypermethylation. Our data nonetheless suggest shared prenatal influences on HIF3A methylation and adiposity.
\end{abstract}

Keywords: birth weight $\bullet$ DNA methylation $\bullet$ embryonic and fetal development

- epigenomics $\bullet$ HIF3A protein $\bullet$ human • obesity

DNA methylation states at particular loci have been associated with a range of disease states and environmental exposures. It is desirable to know whether particular DNA methylation changes occur before disease symptoms or after as a consequence of the disease, as epigenetic biomarkers have been suggested as a way of tracking developmental trajectories to disease [1].

A large epigenome-wide association study (EWAS) with replication in two independent cohorts reported that methylation levels at three CpGs in the first intron of the hypoxia inducible factor 3A (HIF3A) locus were positively associated with BMI in adult Caucasian whole blood and adipose tissue [2]. The finding has since been independently replicated [3]. The authors postulated that HIF3A has a role in acquired obesity, perhaps in regulating adipocyte differentiation.

There is considerable evidence of developmental pathways to obesity [4-7], beginning before birth and at least partly mediated by epigenetics [8-10]. Factors acting prenatally may affect methylation levels at particular loci, which in turn affect transcription and phenotype later in life [1]. Birth weight is often used as a surrogate for the quality of the in utero environment and both low and high birth weights are associated with an increased risk of obesity and metabolic syndrome [11,12]. An association of birth weight or neonatal adiposity with HIF3A methylation at birth would be compatible with the developmental origins of obesity hypothesis. The effects of the prenatal environment on the epigenome could be genotype dependent [13] as we have previously found that umbilical cord methylation levels can associate with various in utero environmental factors in interaction with infant genotype [14]. HIF3A methylation was shown to associate with cis-genotype but the genotype was not associated with adult BMI [2].

The present study has three objectives. First, we tested if HIF3A gene methylation levels in umbilical cords were associated with birth size (weight and length) and adiposity. Second, due to the strong association between
Epigenomics

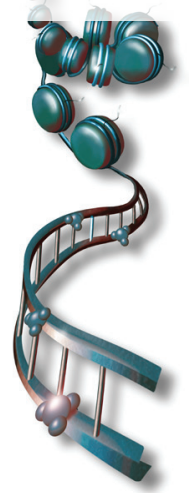

Hong Pan ${ }^{\ddagger}$ Xinyi Lin Yonghui Wu, Li Chen, Ai Ling Teh, Shu E Soh, Yung Seng Lee, Mya Thway Tint, Julia L Maclsaac, Alexander M Morin, Kok Hian Tan, Fabian Yap, Seang Mei Saw, Michael S Kobor, Michael J Meaney, Keith M Godfrey, Yap-Seng Chong, Peter D Gluckman, Neerja Karnani*, Joanna D Holbrook** \& GUSTO Study Group *Author for correspondence: Tel.: +65 64074041 neerja_karnani@sics.a-star.edu.sg **Author for correspondence: Tel.: +447802746050 joanna_holbrook@sics.a-star.edu.sg ${ }^{\ddagger}$ Authors contributed equally For a full list of affiliations please see page 949

Future
Medicine
part of 
cis-SNPs and methylation within the HIF3A locus, we studied if the association of methylation with birth weight is independent of cis-genotype. Third, we sought to identify prenatal environmental variables associated with both birth weight and HIF3A methylation.

\section{Materials \& methods}

Study population

Mother-offspring pairs in our study were drawn from the Growing Up in Singapore towards Healthy Outcomes (GUSTO) birth cohort [15]. Women aged at

\begin{tabular}{|c|c|c|c|}
\hline Characteristic & Time point & $n(\%)$ & Mean (standard deviation) \\
\hline Ethnicity: & Delivery & & \\
\hline Chinese & & $568(57)$ & \\
\hline Malay & & $251(25)$ & \\
\hline Indian & & $172(17)$ & \\
\hline \multicolumn{4}{|l|}{ Child sex: } \\
\hline Male & & $530(53)$ & \\
\hline Female & & $461(47)$ & \\
\hline Gestational age (weeks) & & & $38.9(1.0)$ \\
\hline \multirow[t]{5}{*}{ Weight (g) } & Delivery & 961 & 3150 (376) \\
\hline & 6 months & 872 & 7744 (919) \\
\hline & 12 months & 848 & 9405 (1073) \\
\hline & 18 months & 808 & $10,770(1304)$ \\
\hline & 24 months & 820 & $11,996(1542)$ \\
\hline \multirow[t]{5}{*}{ Length $(\mathrm{cm})$} & Delivery & 961 & $49(2)$ \\
\hline & 6 months & 876 & $67(3)$ \\
\hline & 12 months & 850 & $75(3)$ \\
\hline & 18 months & 695 & $82(3)$ \\
\hline & 24 months & 720 & $88(4)$ \\
\hline \multirow[t]{5}{*}{ BMI $\left(\mathrm{g} / \mathrm{cm}^{2}\right)$} & Delivery & 961 & $1.3(0.1)$ \\
\hline & 6 months & 872 & $1.7(0.2)$ \\
\hline & 12 months & 847 & $1.6(0.1)$ \\
\hline & 18 months & 693 & $1.6(0.1)$ \\
\hline & 24 months & 720 & $1.6(0.1)$ \\
\hline \multirow[t]{3}{*}{ Subscapular skinfold (mm) } & Delivery & 962 & $5.0(1.2)$ \\
\hline & 18 months & 673 & $6.4(1.4)$ \\
\hline & 24 months & 760 & $6.4(1.6)$ \\
\hline \multirow[t]{3}{*}{ Triceps skinfold (mm) } & Delivery & 963 & $5.5(1.2)$ \\
\hline & 18 months & 714 & $8.6(1.7)$ \\
\hline & 24 months & 734 & $8.8(1.8)$ \\
\hline \multirow[t]{3}{*}{ Subscapular: triceps } & Delivery & 962 & $0.93(0.17)$ \\
\hline & 18 months & 648 & $0.76(0.14)$ \\
\hline & 24 months & 723 & $0.73(0.14)$ \\
\hline Methylation of cg27146050 & Delivery & 991 & $15 \%(4 \%)$ \\
\hline Methylation of cg16672562 & & & $19 \%(5 \%)$ \\
\hline Methylation of cg22891070 & & & $31 \%(6 \%)$ \\
\hline rs8102595 minor allele frequency (G) & & & 0.22 \\
\hline rs3826795 minor allele frequency (T) & & & 0.41 \\
\hline
\end{tabular}


Table 2. Association between umbilical cord methylation at three sites in HIF3A and six neonatal anthropometric outcomes (weight, length, BMI, subscapular skinfold, triceps skinfold and ratio of subscapular to triceps skinfold) at birth.

\begin{tabular}{|c|c|c|c|c|c|c|c|c|c|}
\hline & \multicolumn{3}{|c|}{$\operatorname{cg} 27146050$} & \multicolumn{3}{|c|}{ cg16672562 } & \multicolumn{3}{|c|}{$\operatorname{cg} 22891070$} \\
\hline & Est. & $95 \% \mathrm{Cl}$ & p-value & Est. & $95 \% \mathrm{Cl}$ & $p$-value & Est. & $95 \% \mathrm{Cl}$ & $p$-value \\
\hline Birth weight (g) & 3.61 & $(0.68-6.63)$ & 0.015 & 3.34 & $(1.4-5.32)$ & 0.00068 & 2.05 & $(0.32-3.82)$ & 0.020 \\
\hline BMI at birth $\left(\mathrm{g} / \mathrm{cm}^{2}\right)$ & 2.38 & $(0.23-4.58)$ & 0.030 & 2.4 & $(0.97-3.84)$ & 0.00096 & 1.35 & $(0.07-2.64)$ & 0.039 \\
\hline $\begin{array}{l}\text { Subscapular skinfold } \\
\text { at birth }(\mathrm{mm})\end{array}$ & -0.77 & $(-5.97-4.72)$ & 0.78 & 5.44 & $(1.76-9.25)$ & 0.0035 & 3.27 & $(0-6.64)$ & 0.050 \\
\hline $\begin{array}{l}\text { Subscapular: triceps } \\
\text { at birth }\end{array}$ & 0.50 & $(-3.85-5.04)$ & 0.83 & 4.67 & $(1.66-7.77)$ & 0.0022 & 3.02 & $(0.34-5.78)$ & 0.027 \\
\hline
\end{tabular}

least 18 years were prospectively recruited from the KK Women's and Children's Hospital (KKH) and the National University Hospital (NUH) in Singapore during their first trimester of pregnancy. Written informed consent was obtained. The GUSTO study was approved by the ethics boards of both $\mathrm{KKH}$ and NUH. To be eligible, women had to hold Singapore citizenship or permanent residency, intended to reside in Singapore for the next 5 years, were of Chinese, Malay or Indian ethnicity, had homogeneous parental ethnic background and had the intention of delivering at either NUH or KKH. Women who were on chemotherapy, psychotropic drugs or had diabetes mellitus were ineligible. Interviewer-administered questionnaires were used to obtain demographic, medical and obstetric data to assess eligibility. Multiple pregnancies, preterm and intrauterine growth restriction births were excluded from this analysis. There were 1052 live singleton births after excluding 124 preterm and intrauterine growth restriction births. This analysis used 991 offspring with complete information available on HIF3A methylation at three sites (cg27146050, cg16672562, cg22891070), genotype at two cis-SNPs (rs8102595 and rs3826795) and at least one anthropometric outcome.

\section{Offspring anthropometry}

Offspring weight and recumbent length were measured at birth, 6, 12, 18 and 24 months of age. Weight was measured using a calibrated infant scale (SECA 334 Weighing Scale, SECA Corp) and recorded to the nearest gram. Length was measured using a SECA infant mat (SECA 210 Mobile Measuring Mat, SECA Corp) and recorded to the nearest $0.1 \mathrm{~cm}$. Measurements were taken in duplicates for reliability. BMI was derived as weight $(\mathrm{g})$ divided by length ${ }^{2}\left(\mathrm{~cm}^{2}\right)$ at all time points. Subscapular and triceps skinfolds were measured at birth, 18 and 24 months and taken in triplicates using Holtain skinfold calipers (Holtain Ltd, Crymych, UK) on the right side of the body, recorded to the nearest $0.2 \mathrm{~mm}$. Subscapular to triceps skinfold ratio was derived by dividing subscapular skinfold $(\mathrm{mm})$ by triceps skinfold (mm).

\section{Prenatal environmental exposures}

An interviewer-administered questionnaire was conducted at 26-28 weeks of gestation to obtain information on occupational activity during pregnancy, exercise during pregnancy, alcohol usage before and during pregnancy and smoking patterns before and during pregnancy (Supplementary File 1; for supplementary information please see online at: www.futuremedicine.com/doi/ full/10.2217/EPI.15.45). Maternal height and weight were measured during the same time period. Prepregnancy weight was self-reported during study recruitment in the first trimester of pregnancy. Pregnancy weight gain was calculated as the difference between prepregnancy and 26-28-week weights. Maternal prepregnancy BMI was derived as prepregnancy weight divided by height squared. Maternal glucose levels, indicative of gestational diabetes, ( $2 \mathrm{~h}$ postglucose and fasting) were ascertained at 26-28 weeks using an oral glucose tolerance test (OGTT) of $75 \mathrm{~g}$ after overnight fasting (8-10 h). Information on maternal glucose levels ( $2 \mathrm{~h}$ postglucose and fasting) were available for 922 subjects. Using the 1999 WHO standard criteria $(\geq 7.8 \mathrm{mmol} / \mathrm{l}$ for $2-\mathrm{h}$ postglucose and/or $\geq 7.0 \mathrm{mmol} / 1$ for fasting glucose), 162 out of 922 subjects would be diagnosed as having ges- 


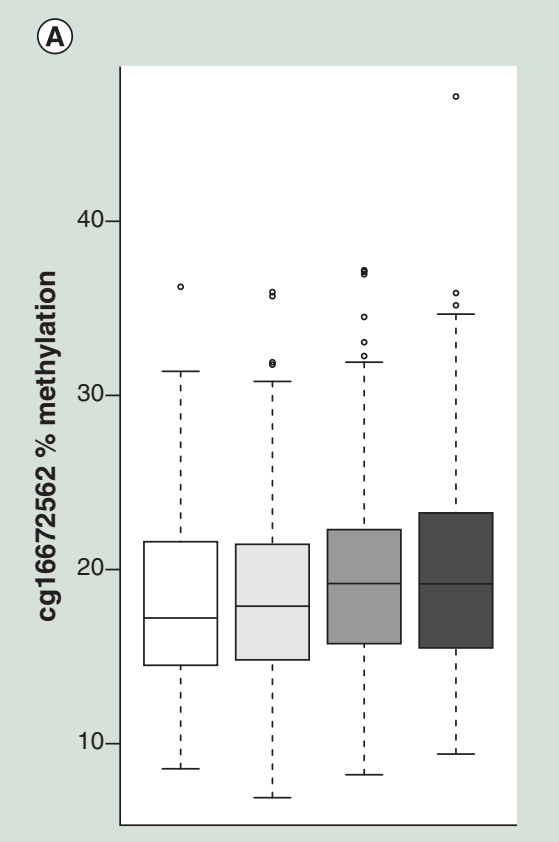

Birth weight (g)
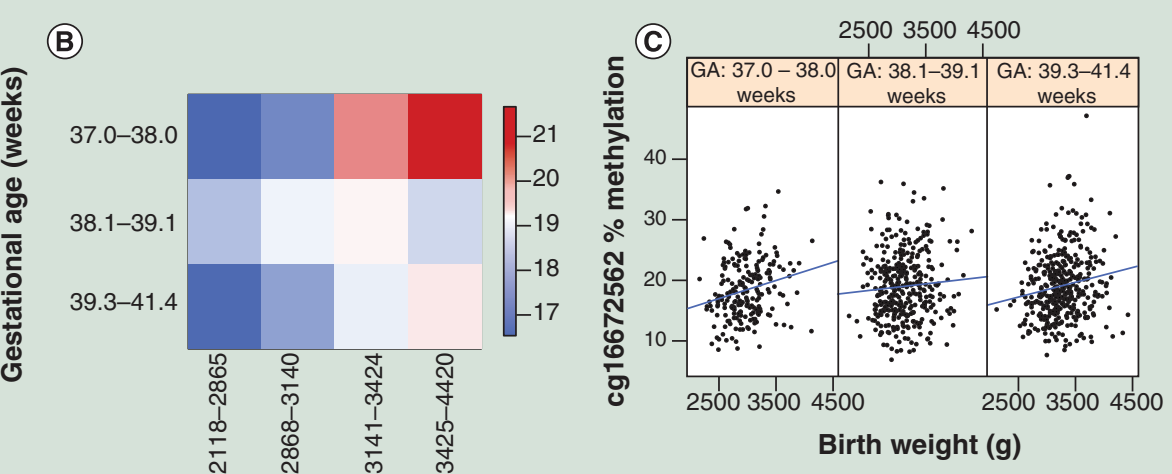

(D)

Birth weight (g)
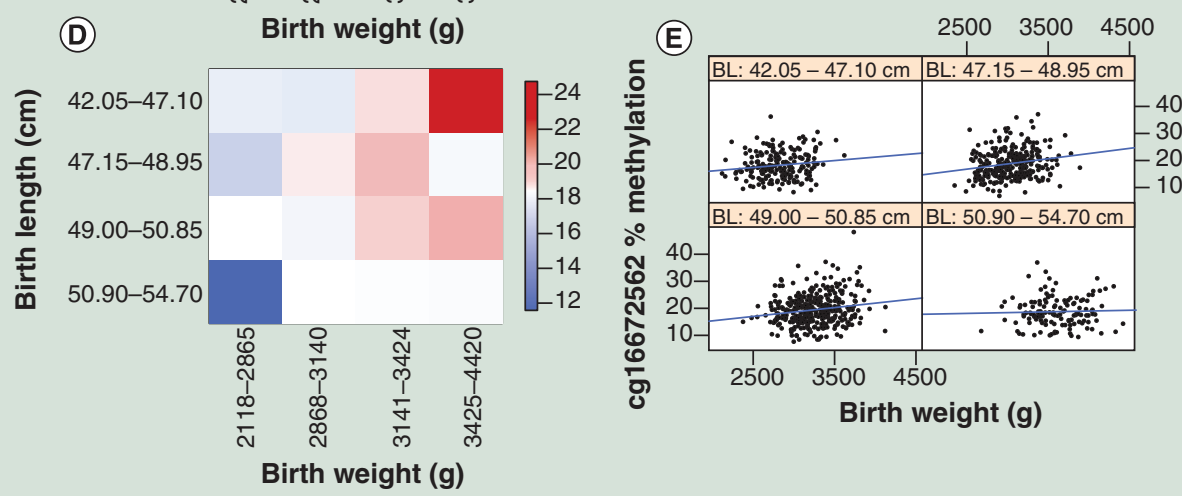

$\begin{array}{lllll}\text { Birth weight (g) } & 2118-2865 & 2868-3140 & 3141-3424 & 3425-4420 \\ \text { Count } & 231 & 253 & 266 & 211 \\ \text { Median methylation (\%) } & 17.2 & 17.9 & 19.2 & 19.2 \\ \text { Mean methylation (\%) } & 18.0 & 18.4 & 19.4 & 19.9\end{array}$

Figure 1. Association between percentage methylation at cg16672562 and birth weight. (A) Box plot of birth weight (horizontal axis) binned into four bins of equal numbers of unique values, against \% methylation at cg16672562 (vertical axis). Number of individual data points, median and mean \% methylation of each bin is displayed in the table below the horizontal axis. (B) Heatmap displaying $\%$ methylation at cg16672562, maximum, medium and minimum methylation are shown in red, white and blue, respectively. Subjects are split by binned (four bins of equal number of unique values) birth weight (horizontal axis) and binned (four bins of equal number of unique values, last two bins are combined as there are few observations in the last bin) gestational age (vertical axis). The progression from blue to red (low to high methylation) as birth weight increases (left to right) is visible in each gestational age bin (top to bottom). (C) Scatter plots of birth weight (horizontal axis) against \% methylation at cg16672562 (vertical axis), each panel displays data for binned gestational ages (four bins of equal number of unique values, last two bins are combined as there are few observations in the last bin), ranges for each bin are displayed in panel headers. (D) Heatmap displaying \% methylation at cg16672562, maximum, medium and minimum methylation are shown in red, white and blue, respectively. Subjects are split by binned (four bins of equal number of unique values) birth weight (horizontal axis) and binned (four bins of equal number of unique values) birth length (vertical axis). The progression from blue to red (low to high methylation) as birth weight increases (left to right) is visible in each birth length bin (top to bottom). (E) Scatter plots of birth weight (horizontal axis) against \% methylation at cg16672562 (vertical axis), each panel displays data for binned (four bins of equal number of unique values) birth length, ranges for each bin are displayed in panel headers.

For color images please seen online at: www.futuremedicine.com/doi/full/10.2217/EPI.15.45

tational diabetes in this group. Maternal plasma micronutrient levels, including vitamin D, vitamin B12, copper, iron, zinc and folate, were tested using serum drawn at 26-28 weeks of gestation. Birth order and mode of delivery were abstracted from hospitals' medical records.

\section{Methylation \& genotyping data}

Bisulfite-converted gDNA from umbilical cords was interrogated on Infinium ${ }^{\circledR}$ Human Methylation 450
Bead Chip arrays [16] as per manufacturer's instructions and as described previously [14]. Data were processed as previously described [17]. Briefly, signal extraction was performed in GenomeStudio ${ }^{\mathrm{TM}}$ Methylation Module and raw values were extracted. Probes with data from two beads or fewer for any sample, or with signal detection p-values (calculated from signal vs background of the individual bead intensities) greater than 0.01 , for any sample, were discarded for all samples. The green 
Table 3. Association of two cis-single nucleotide polymorphisms (rs8102595, rs3826795) with methylation at three sites in HIF3A.

\begin{tabular}{|c|c|c|c|c|c|c|c|c|c|}
\hline \multirow[t]{2}{*}{ Model } & \multicolumn{3}{|c|}{$\operatorname{cg} 27146050$} & \multicolumn{3}{|c|}{ cg16672562 } & \multicolumn{3}{|c|}{ cg22891070 } \\
\hline & Est. & $95 \% \mathrm{Cl}$ & $p$-value & Est. & $95 \% \mathrm{Cl}$ & $p$-value & Est. & $95 \% \mathrm{Cl}$ & $p$-value \\
\hline CpG rs8102595 & 1.2 & $(0.96-1.5)$ & 5.7E-19 & 2.9 & $(2.5-3.3)$ & $1.6 \mathrm{E}-45$ & 3.2 & $(2.8-3.7)$ & 5.7E-47 \\
\hline \multicolumn{10}{|c|}{$\begin{array}{l}\text { Above are results from six different linear regression models. In each linear regression model, methylation at each CpG site is regressed against each SNP. Regression } \\
\text { coefficients (Est.) and } 95 \% \text { CI reflect changes in \% methylation per copy of the effect allele, adjusting for child sex, ethnicity, cell type proportions and interactions } \\
\text { between ethnicity and cell type proportions. p-values are two-sided and p-values less than } 0.05 \text { are shown in bold. The effect allele of rs } 8102595 \text { is } \mathrm{G} \text { and the effect } \\
\text { allele of rs3826795 is C. }\end{array}$} \\
\hline
\end{tabular}

and red channel signals were normalized and the background signal from the negative probe control values was removed. Probe $\beta$-values or $\%$ methylation values were derived from the data. $\beta$-values are the ratio of the methylated probe intensity and the overall intensity, $\beta$-value for an $i$ th interrogated $\mathrm{CpG}$ site:

$$
\beta_{\mathrm{i}}=\frac{\max \left(\mathrm{Yi}_{\mathrm{i}, \text { methy, }} 0\right)}{\max \left(\mathrm{Y}_{\mathrm{i}, \text { unmechy, }} 0\right)+\max \left(\mathrm{Y}_{\mathrm{i}, \text { methy, }} 0\right)+\mathrm{a}}
$$

Equation 1

where $\mathrm{y}_{\mathrm{i} \text {,methy }}$ and $\mathrm{y}_{\mathrm{i} \text {,unmethy }}$ are the intensities measured by the $i$ th methylated and unmethylated probes, respectively, averaged over the replicate beads; $\mathrm{a}$ is a constant offset, which is by default 100 . Therefore, $\beta$-values range between 0 and 1 with 0 representing no methylation and 1 representing $100 \%$ methylation. $\beta$-values were further processed to scale the $\%$ methylation range of the type 2 probes to the type 1 probes using the procedure suggested by [18]. All data from the sex chromosomes were removed and the remaining data were subjected to quantile normalization. As part of the experimental design, subjects were randomized such that key variables (birth weight, child sex, ethnicity, gestational age) were randomly distributed across batch/chip/position. Batch effects were observed between different runs in the processed data and removed by a commonly used empirical Bayes method known as COMBAT [19]. Even though chip/position were associated with the three $H I F 3 A$ methylation sites in question (Supplementary Table 1), chip/position were not associated with any of the six neonatal outcomes studied in this report (Supplementary Table 2) due to experimental design. As chip/position were associated only with the independent variable (methylation) but not the outcome, this minimizes confounding bias due to chip/position.

The same umbilical cord DNA samples were genotyped using the Illumina Omniexpress + exome arrays. Genotyping was performed by the service provider, Expression Analysis Inc. Data were processed in Genome Studio Genotyping Module ${ }^{\mathrm{TM}}$. Genotyping calls were made by the Gen Call software, which incorporates a clustering algorithm (GenTrain) and a calling algorithm (Bayesian model). Gen Call score of each single nucleotide polymorphism (SNP) probe and call rate of each sample are generated. Genotypes with a Gen Call score less than 0.15 were not considered.

\section{Accounting for cellular heterogeneity from methylation data}

Umbilical cord tissue consists of a mixture of cell types with differing epigenetic profiles. To control for cellular heterogeneity in our samples, we applied three methods: one requiring a reference panel of methylation levels in discrete cell types [20] and two referencefree methodologies [21,22]. For the former, we estimated the proportion of fibroblasts, B cells and T cells using a reference panel's cell-specific methylation profiles [20]. The methylation dataset obtained from EMBL-EBI European Genome-Phenome Archive [23] under accession number EGAD00010000460 was used as our reference panel [24]. The estimated cell fractions were then adjusted as covariates in the regression models. Interestingly, the associations between the estimated cellular

Table 4. Association between two cis-single nucleotide polymorphisms at the HIF3A locus (rs8102595, rs3826795) and birth weight.

\begin{tabular}{|llll}
\hline Single nucleotide polymorphisms & Est. & $\mathbf{9 5 \% ~ C l}$ & p-value \\
rs8102595 & 0.39 & $\mathbf{( - 0 . 8 9 - 1 . 7 )}$ & 0.55 \\
\hline rs3826795 & 0.059 & $\mathbf{( - 1 . 0 - 1 . 2 )}$ & 0.92 \\
\hline $\begin{array}{l}\text { Regression coefficients (Est.) and 95\% Cl are reported as percentage change in birth weight per copy of the effect allele. p-values are } \\
\text { two-sided. Analysis was done by linear regression of log-transformed birth weight against each SNP (additive genetic model), adjusting for } \\
\text { ethnicity and child sex. The effect allele of rs8102595 is G and the effect allele of rs3826795 is C. }\end{array}$ \\
\hline
\end{tabular}


Table 5. Association between umbilical cord methylation at three sites in HIF3A and six neonatal anthropometric outcomes (weight, length, BMI, subscapular skinfold, triceps skinfold and ratio of subscapular to triceps skinfold) at birth, adjusting for rs8102595 and rs3826795

\begin{tabular}{|c|c|c|c|c|c|c|c|c|c|}
\hline \multirow[t]{2}{*}{ Outcome } & \multicolumn{3}{|c|}{$\operatorname{cg} 27146050$} & \multicolumn{3}{|c|}{ cg16672562 } & \multicolumn{3}{|c|}{ cg22891070 } \\
\hline & Est. & $95 \% \mathrm{Cl}$ & p-value & Est. & $95 \% \mathrm{Cl}$ & p-value & Est. & $95 \% \mathrm{Cl}$ & $p$-value \\
\hline Birth weight (g) & 3.67 & $(0.62-6.81)$ & 0.018 & 3.87 & $(1.7-6.09)$ & 0.00044 & 2.28 & $(0.34-4.26)$ & 0.021 \\
\hline Birth length $(\mathrm{cm})$ & 0.63 & $(-0.3-1.57)$ & 0.18 & 0.55 & $(-0.11-1.21)$ & 0.10 & 0.4 & $(-0.19-1)$ & 0.18 \\
\hline BMI at birth $\left(\mathrm{g} / \mathrm{cm}^{2}\right)$ & 2.37 & $(0.13-4.67)$ & 0.038 & 2.74 & $(1.14-4.36)$ & 0.00075 & 1.46 & $(0.03-2.91)$ & 0.046 \\
\hline $\begin{array}{l}\text { Subscapular skinfold at } \\
\text { birth (mm) }\end{array}$ & -0.62 & $(-6.05-5.11)$ & 0.83 & 7.08 & $(2.92-11.41)$ & 0.00073 & 4.31 & $(0.64-8.11)$ & 0.021 \\
\hline $\begin{array}{l}\text { Triceps skinfold at birth } \\
(\mathrm{mm})\end{array}$ & -0.06 & $(-5.46-5.65)$ & 0.98 & 2.64 & $(-1.33-6.77)$ & 0.19 & 1.7 & $(-1.85-5.39)$ & 0.35 \\
\hline $\begin{array}{l}\text { Subscapular: triceps } \\
\text { at birth }\end{array}$ & -0.6 & $(-5.06-4.08)$ & 0.8 & 4.34 & $(1-7.79)$ & 0.011 & 2.57 & $(-0.4-5.63)$ & 0.091 \\
\hline
\end{tabular}

proportions and child anthropometry were generally ethnic-dependent (Supplementary Tables 3 \& 4). Therefore, interaction terms between cellular proportions and ethnicity were included in all regression models. As a sensitivity analysis, we also applied two reference-free methods $[21,22]$ to investigate the association between methylation levels at HIF3A and log-transformed child anthropometric measures, adjusting for infant sex and ethnicity. Leek et al. [22] estimates surrogate variables which capture cellular heterogeneity, the surrogate variables are then adjusted as covariates in regression analysis. We note that this sensitivity analysis using Leek et al. [22] would also act to adjust for residual unknown confounding effects, including chip/position effects since the estimated surrogate variables can potentially capture both cellular heterogeneity and residual batch/chip/position effects [21,2527]. The method proposed by Houseman et al. [21] is similar to the surrogate variable analysis proposed by Leek et al. [22] with an additional biological mixing assumption. Houseman et al. [21] correct the cellular heterogeneity without explicitly estimating the relative cell proportions or returning the surrogate variables.

\section{Statistical analysis}

Linear regression models were used to examine the association of child anthropometric measures with HIF3A methylation $\beta$-values, with adjustment for child sex, ethnicity, cellular composition and interactions between ethnicity and cellular proportions. Child anthropometric measures were log-transformed to satisfy statistical assumptions of normality. Due to the logtransformation, effect sizes are reported as percentage increase in child outcome for $10 \%$ increase in methylation $\beta$-values. As a sensitivity analysis, we also report results additionally adjusted for gestational age. To examine if the association between child anthropometry with HIF3A methylation were independent of cis-genotypes, we additionally adjusted for both cis-genotypes. To identify prenatal environmental exposures associated with both birth weight and HIF3A methylation, we regressed birth weight on various prenatal exposures, and adjusted for child sex and ethnicity. We further regressed HIF3A methylation on various prenatal exposures (and their interactions with genotype), adjusting for child sex, ethnicity, cellular composition and interactions between ethnicity and cellular proportions.

Table 6. Two-sided $p$-values from testing for interaction term between log-transformed birth weight and single nucleotide polymorphism (rs8102595, rs3826795), with methylation as outcome, adjusting for main effect of log-transformed birth weight, main effect of single nucleotide polymorphism, child sex, ethnicity, cell type proportions and interactions between ethnicity and cell type proportions.

\begin{tabular}{|llll|}
\hline Model & cg27146050 & cg16672562 & cg22891070 \\
& p-value & p-value & p-value \\
$\log (B W) *$ rs8102595 & 0.32 & 0.077 & 0.30 \\
$\log (B W) *$ rs3826795 & 0.34 & 0.019 & 0.033 \\
\hline
\end{tabular}



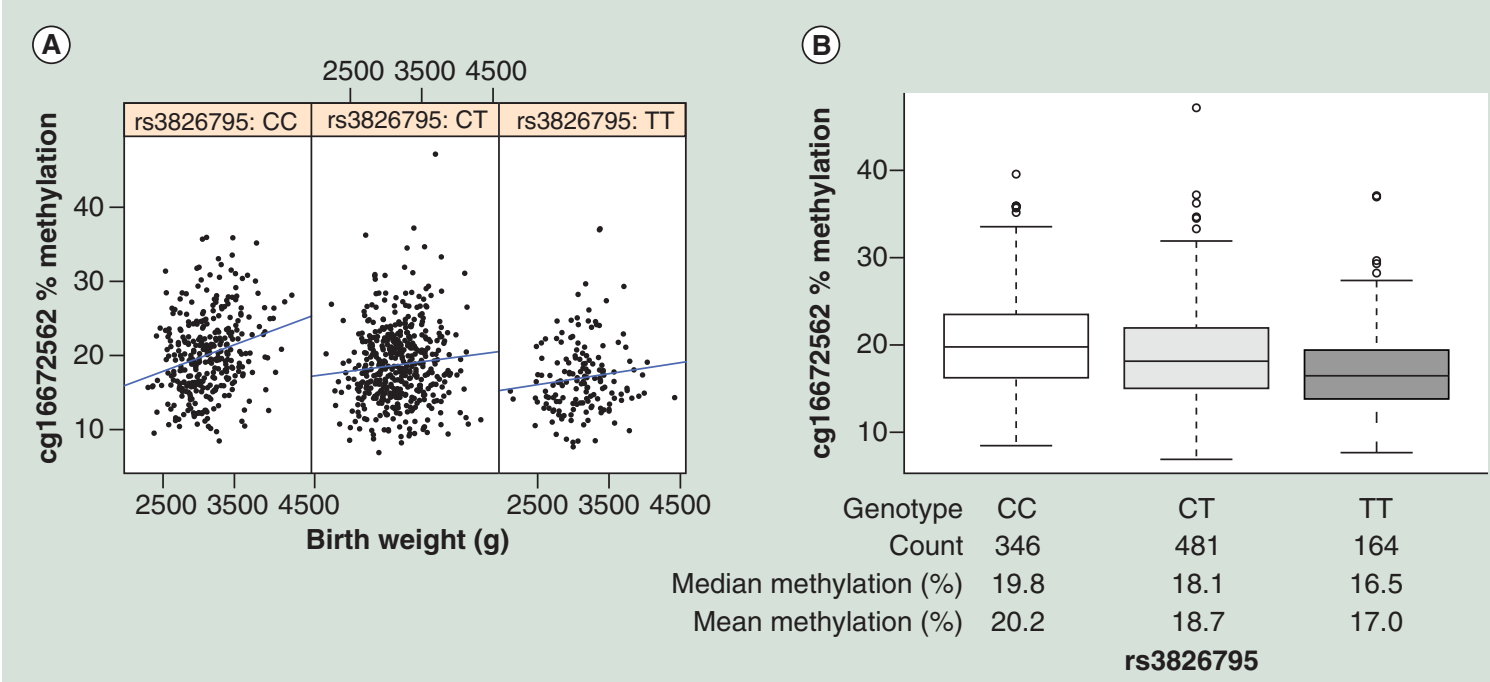

Figure 2. Association between \% methylation at cg16672562 and birth weight, stratified by rs3826795 genotype. (A) Scatter plots of birth weight (horizontal axis) against \% methylation at cg16672562 (vertical axis), each panel displays data for each genotype. (B) Box plot of rs3826795 genotype (horizontal axis) against \% methylation at cg16672562 (vertical axis). Number of individual data points, median and mean \% methylation of each bin is displayed in the table below the horizontal axis.

\section{Results}

HIF3A methylation in umbilical cords was positively associated with birth weight \& adiposity

Table 1 gives the characteristics of the 991 infants investigated in the current analysis. Higher umbilical cord HIF3A methylation level at all three CpGs was associated with greater infant birth weight (Table 2 \& Figure 1A), after adjusting for infant sex, ethnicity, estimated cellular proportions and interactions between ethnicity and cellular proportions [20]. There were also significant associations with adiposity at birth measured by BMI and, for two of the three CpGs, by subscapular skinfold and the ratio of subscapular to triceps skinfold (thought to reflect fat deposition on the trunk rather than limbs [28]) (Table 2). Similar results were obtained at two of the CpGs when we used reference-free methodologies $[21,22]$ to account for cellular heterogeneity (Supplementary Tables 5 \& 6). Despite the strong association between gestational age and birth weight in our dataset $(\mathrm{r}=0.32 ; \mathrm{p}<0.0005)$, the associations of HIF3A methylation at all three CpGs with birth weight remained significant, after adjusting for gestational age (Supplementary Table 7). The association between methylation and birth weight was consistent across gestational age categories and was perhaps strongest in neonates with the earliest and latest gestational ages (Figure 1B \& C \& Supplementary Table 8). Similarly, despite the strong correlation between birth weight and length in the dataset $(\mathrm{r}=0.67 ; \mathrm{p}<0.0005)$, HIF3A methylation was not significantly associated with birth length and the association between methylation and birth weight was consistent across birth length categories (Figure 1D \& E), suggesting a link between HIF3A methylation and adiposity at birth rather than birth size in general.

For every $10 \%$ increase in methylation of $\operatorname{cg} 27146050, \operatorname{cg} 16672562$ and $\operatorname{cg} 22891070$, birth weight was $3.61 \%(95 \%$ CI: $0.68-6.63), 3.34 \%$ (1.4-5.32) and $2.05 \%(0.32-3.82)$ higher, respectively (Table 2). These effect sizes are slightly smaller than the corresponding effect estimates on adult BMI of 7.8\% $(5.1-10.4), 3.2 \%(2.0-4.4)$ and $3.6 \%(2.4-4.9)$, as previously reported [2].

The association of HIF3A methylation \& birth weight remained significant after adjusting for cis-acting SNPs; but birth weight \& HIF3A methylation was more strongly correlated in one genotypic group

Using the same 991 GUSTO umbilical cord samples, we genotyped the SNPs identified as cis-influencing HIF3A methylation in the Dick et al.'s study [2]. We found that rs8102595 and rs3826795 were strongly associated with methylation levels at all three CpGs sites (Table 3). However, neither SNP was associated with birth weight (Table 4) and the associations of birth weight with methylation at all three sites were similar when adjusted for genotype at both SNPs (Table 5). This mirrors the findings of [2]. We also tested if HIF3A methylation levels were explained by an interaction of HIF3A genotype and infant birth weight (adjusting for 
(i) Environment independently affects both HIF3A methylation and adiposity

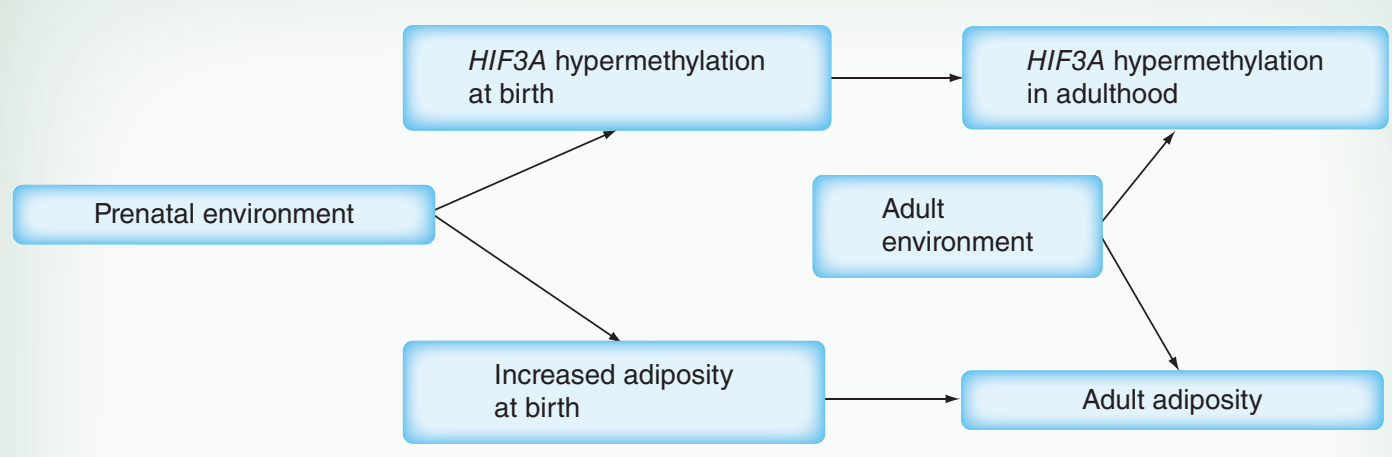

(ii) HIF3A methylation affects adiposity

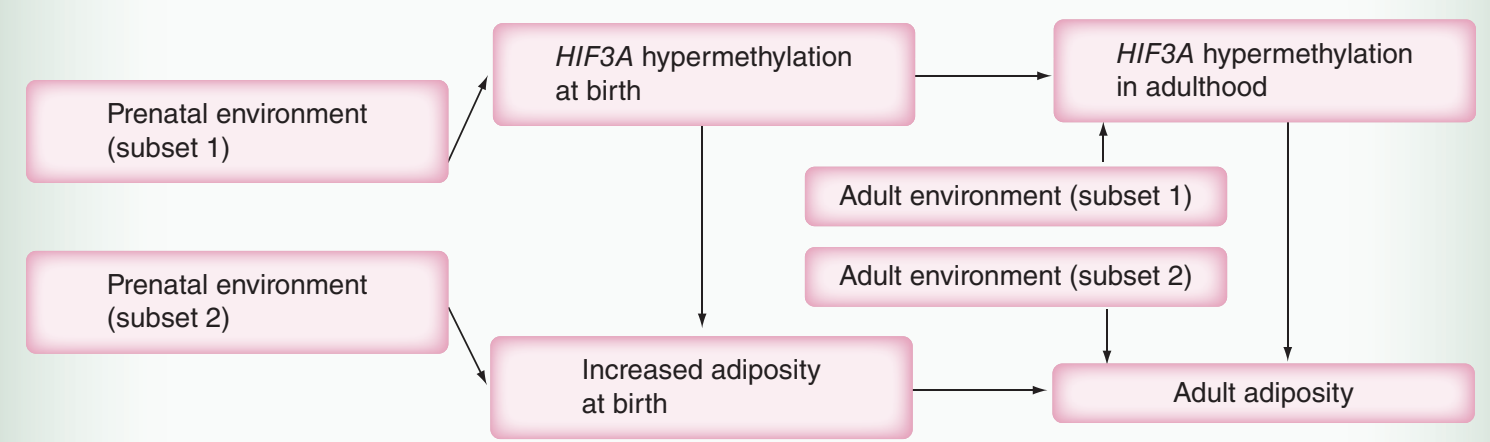

(iii) Adiposity affects HIF3A methylation

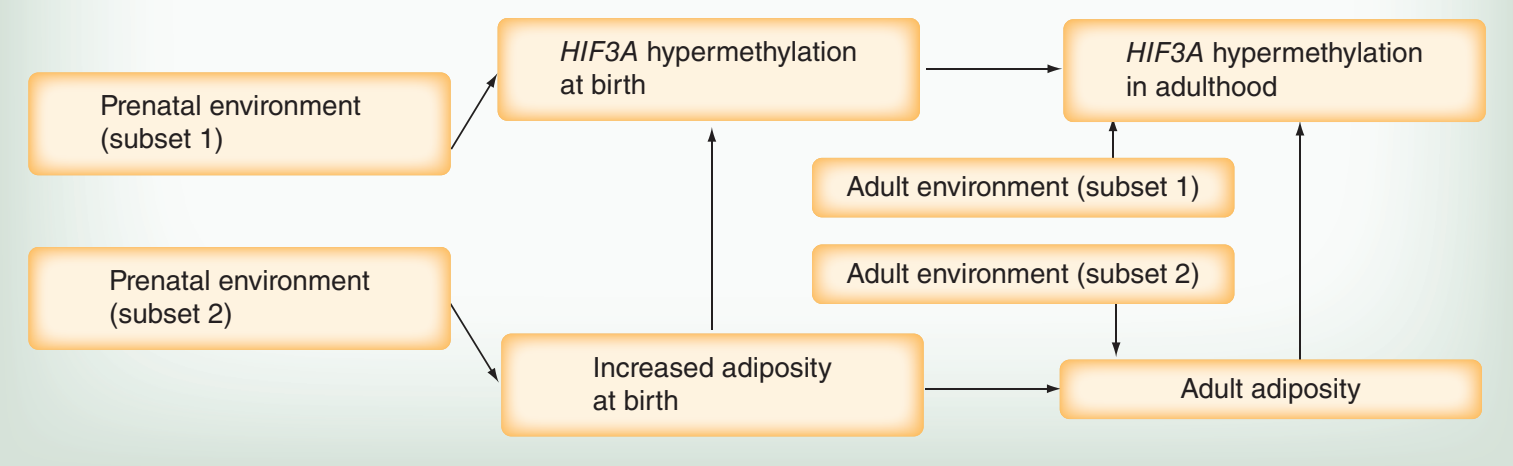

Figure 3. Three of the simplest possible scenarios to explain the relationship between HIF3A methylation and adiposity.

main effects of both genotype and infant birth weight), as we have previously found that neonatal methylation patterns are often a product of the interaction of the in utero environment and genotype. The interaction term was significant at $\mathrm{p}<0.05$ for two of the CpGs and rs3826795 (Table 6). In the CC genotype group, birth weight was more strongly correlated with HIF3A methylation (Figure 2 \& Supplementary Table 9). The CC genotype was also associated with higher methylation values at the HIF3A locus (Table 3 \& Figure 2). This suggests the CC genotypic group could be more plastic to environmental exposures in utero.

The prenatal environmental variables examined were not consistently associated with HIF3A methylation \& birth weight

As we hypothesized that HIF3A methylation and birth weight were related to the prenatal environment, we examined for significant associations with both HIF3A methylation and birth weight for a range of factors 
related to the prenatal environment, both as main effects and in interaction with $H I F 3 A$ genotype. Factors investigated included antenatal lifestyle factors such as activity level and smoking, maternal prepregnancy BMI and pregnancy weight gain, maternal glucose tolerance during pregnancy indicative of gestational diabetes, maternal micronutrient levels, maternal age and parity (Supplementary File 1). There were some suggestive associations; for example, maternal glucose tolerance (indicative of gestational diabetes) was significantly associated with both HIF3A methylation at one of the $\mathrm{CpGs}$ and birth weight. Furthermore, maternal weight gain during pregnancy was significantly associated both with infant birth weight and HIF3A methylation (again at only one $\mathrm{CpG}$ ) in interaction with genotype. However, we did not find any prenatal environmental variables that showed consistently significant association with all three HIF3A sites and birth weight and none yielded substantial effect sizes.

\section{Discussion}

We have extended the finding that HIF3A DNA methylation in adults associates with BMI [2], to show that the link between HIF3A DNA methylation with weight and adiposity can be detected at birth. Interestingly, the association was limited to measures of adiposity (i.e., weight, BMI and skinfolds) and not other determinants of birth size or putative proxies for gestational

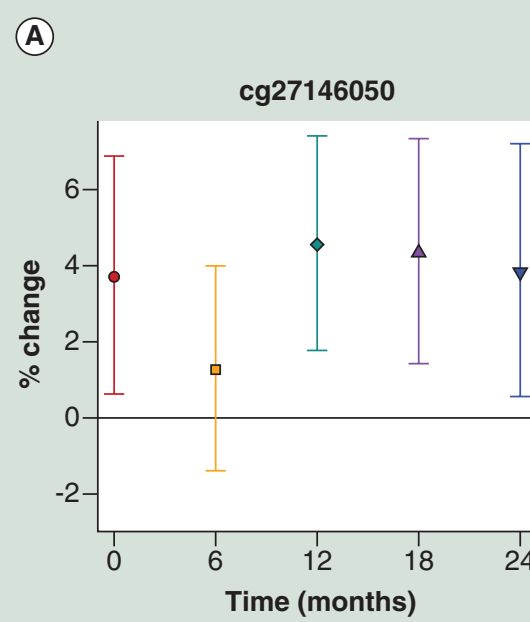

\section{Associations of child weight with:}

cg16672562

cg22891070
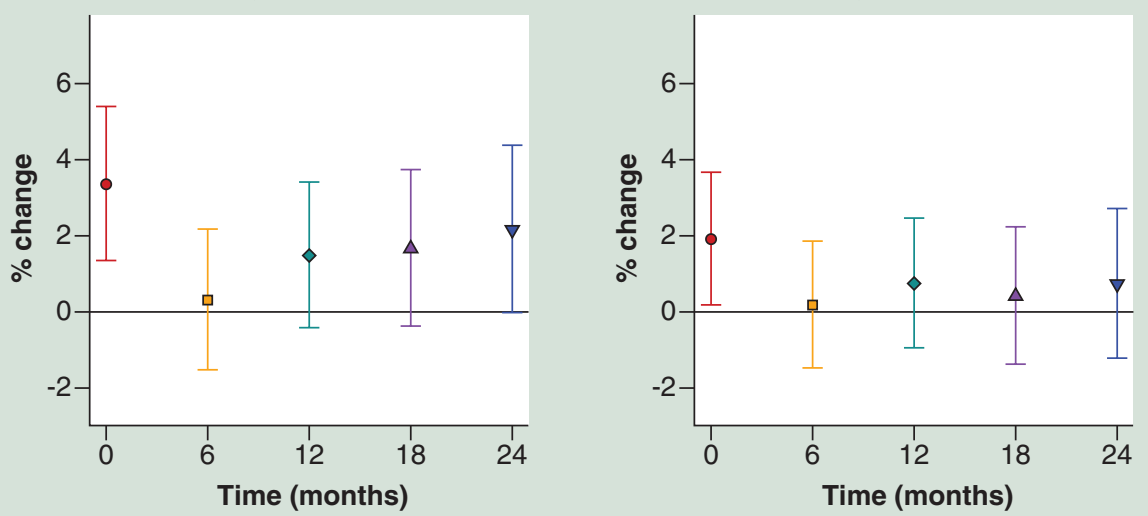

(B)

Associations of child weight, after adjusting for birth weight, with:

$\operatorname{cg} 27146050$

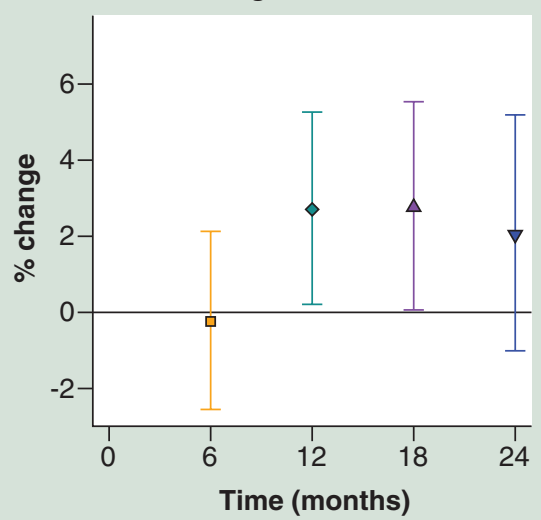

cg16672562

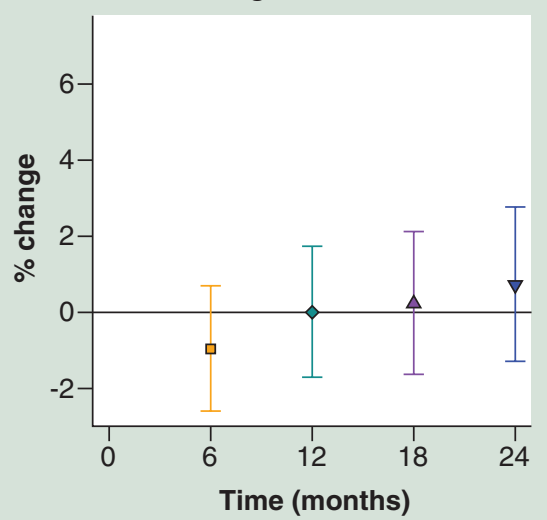

cg22891070

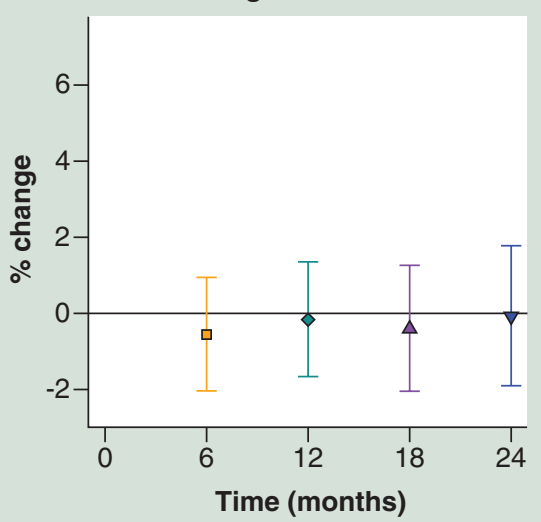

Figure 4. Association between umbilical cord methylation at three sites in HIF3A and child weight measured at 0, 6, 12, 18 and 24 months. (A) Without adjusting for birth weight. (B) Adjusted for log-transformed birth weight. Regression coefficients (Est.) and $95 \% \mathrm{Cl}$ are reported as percentage change in child weight for $10 \%$ increase in methylation level. P-values are two-sided. Using linear regression models estimated using generalized estimating equations to account for repeated measures, we regressed logtransformed weight against methylation at each CpG site, adjusting for fixed effects of time, child sex, ethnicity, cell type proportions and interactions between ethnicity and proportions. Time was coded using a binary variable for each distinct time point and interaction terms of time with all variables (methylation, child sex, ethnicity, cell type proportions and interactions between ethnicity and proportions) were included. 
quality such as gestational age and birth length. As such, the relationship of increased methylation at HIF3A CpGs with birth outcomes is reminiscent of the effect of maternal diseases, like Type 1 diabetes, on the offspring, whereby weight is increased, but length is not [29].

Although pertaining to the same three $\mathrm{CpGs}$ within the HIF3A gene that were previously reported, our data were derived from a different tissue (umbilical cord vs blood and adipose), in a different population (Asian vs Caucasian), in a population-based cohort (normative range vs study population for metabolic disorders and controls) and most importantly at a different stage in the lifecourse (neonates vs adults). The effect size reported here are slightly smaller than those reported by Dick et al. [2]. Reasons the effect sizes are smaller could be statistical, population dependent (our population are healthy babies, Dick et al. studied a disease cohort with a high level of obesity) or developmental (the effect is smaller at birth and increases during the lifecourse as adiposity increases).

Replicated positive associations such as the one described here can guide future hypothesis-free EWAS. Lack of statistical power is a big challenge in EWAS, especially in cohorts of modest size. The integration of effects of cellular heterogeneity, cis and trans genotype and the potential influence of pregnancy conditions is also challenging. Therefore, a detailed case study of a proposed association (HIF3A with adiposity replicated across populations and ages) is important. It will help set the standards for statistical replication and delineate the issues to consider when evaluating hits from more open-ended screening.

Dick et al. [2] considered three possibilities that could explain the association between HIF3A methylation and adiposity in adults: (i) a confounding factor (e.g., environment) independently affects both HIF3A methylation and adiposity, (ii) increased HIF3A methylation causes increased adiposity and (iii) increased adiposity causes increased HIF3A methylation. To put our findings in neonates in context with the findings in adults, the same three simplified possible scenarios are schematically depicted in Figure 3 . We note that more complicated scenarios or combinations of these simplified situations are possible and likely.

Using a Mendelian randomization reasoning [30] from the observation that HIF3A genotype was associated with HIF3A methylation but not adult BMI, Dick et al. [2] suggested that scenario (iii) (adiposity results in HIF3A hypermethylation) is more likely than scenario (ii) (HIF3A hypermethylation leads to increased adiposity). However, in this context, Mendelian randomization assumes the genotype can affect the phenotype only through DNA methylation and not through other biological pathways. The applicability of this assumption is unknown for HIF3A genotype. We cannot confer cau- sality in our dataset but following the same arguments, increased DNA methylation at the HIF3A locus could result from increased birth weight/adiposity and not cause it, but the aforementioned limitation also applies in our case.

Dick et al. considered scenario (i) less likely as they did not find an association between HIF3A methylation and BMI-related characteristics like diabetes. However, the absence of an association between HIF3A methylation and diabetes could also be due to the same confounding factor having a direct effect on glucose tolerance that is independent of increased adiposity. We postulate that scenario (i) could involve a prenatal environmental factor that independently affected both birth weight and HIF3A methylation. We also find that, as may be expected from other studies [14,31], interindividual variation in HIF3A methylation can be explained by an interaction of genotype and birth weight, suggesting there may be a genotype group (CC) particularly sensitive to gestational environment (Table 6 \& Figure 2). However, in this study, we were unable to definitively identify a factor acting prenatally that is consistently associated with both HIF3A methylation and birth weight, despite the extensive dataset on pregnancy environments collected from the GUSTO cohort and examined in this study. Nevertheless, HIF3A methylation at birth after much further study, may prove to be a useful surrogate for likely metabolic trajectory determined by gestational environment [32], and could be more useful than birth weight as it would be independent of the inherited genetic and environmental determinants of birth weight.

Transgenerational transmission of obesity and the importance of the very early environment in determining metabolic trajectories have been much studied [33,34]. If metabolic trajectory is programmed during gestation, a biomarker of likely trajectory would aid intervention [1]. Birth weight has been shown to predict later weight [35] and is strongly associated with later weight in the GUSTO cohort. Interestingly, HIF3A methylation levels at birth were also associated with weights at later ages, albeit possibly with slightly lower effect sizes (Figure 4A \& Supplementary Table 10). Only a few of the associations survive at borderline significance when adjusted for birth weight (Figure 4B \& Supplementary Table 11), thus the association of HIF3A methylation with later weights may reflect the growth trajectory set at birth. The 'dip' in the effect size at 6 months could be due to the effects of weaning or the decreased influence of genetic factors [36,37], although it is interesting to note that birth weight is more correlated with weight at 6 months than with weight at any of the other time points (Supplementary Tables 12 \& 13). 
Future studies should track both $H I F 3 A$ methylation and offspring size and adiposity longitudinally during gestation, at birth, and at subsequent ages to determine if the association of $H I F 3 A$ methylation with adiposity is consistent. As blood and adipose are invasive tissues to sample in infants, $H I F 3 A$ methylation levels should be investigated in other tissue types such as saliva, buccal swabs or circulating fetal cells obtained prenatally. Longitudinal studies can help determine, if $H I F 3 A$ is a biomarker for obesity risk and assess the utility of using HIF3A methylation to evaluate the efficacy of interventions such as lifestyle modification. Future studies should also examine the impact of $H I F 3 A$ methylation changes on gene expression in relevant tissues. Dick et al. [2] found a negative association between $H I F 3 A$ methylation levels and $H I F 3 A$ gene expression in adipose tissue. It is a limitation of our study that we cannot examine gene expression in a relevant tissue due to the unfeasibility of sampling adipose tissue in neonates or infants.

\section{Conclusion}

The association between increased DNA methylation at $H I F 3 A$ and increased adiposity is present in neonates. This suggests $H I F 3 A$ methylation is a potential biomarker for metabolic trajectory and/or implicated in metabolic syndrome and further study is warranted.

\section{Acknowedgements}

The GUSTO study group includes P Agarwal, A Biswas, CL Bong, BFP Broekman, S Cai, JKY Chan, YH Chan, CYI Chee, HYH Chen, YB Cheung, A Chia, A Chinnadurai, CK Chng, MFF Chong, SC Chong, MC Chua, CM Ding, EA Finkelstein, D Fok, M Fortier, AEN Goh, YTD Goh, JJ Gooley, WM Han,

\section{Executive summary}

\section{Introduction}

- Variation in DNA methylation between individuals is a product of influences from the environment and individual genetic backgrounds.

- DNA methylation levels at particular loci may closely map trajectories to diseases such as obesity, which have environmental and genetic components.

- A recent study linked methylation within the HIF3A gene to BMI and obesity in adults.

- Individual susceptibility to obesity is influenced by early environmental exposures.

- This study investigates whether the association of HIF3A methylation with adiposity can be detected at birth.

Material \& methods

- The study was conducted in the 991 subjects from the GUSTO birth cohort.

- We determined HIF3A genotype and DNA methylation patterns in DNA extracted from umbilical cords of the 991 infants using Illumina ${ }^{\circledR}$ Infinium HumanMethylation and Omniexpress arrays.

- Associations of methylation levels and birth outcomes were adjusted for sex, ethnicity, cellular composition of umbilical cords and interactions between ethnicity and cellular composition.

\section{Results}

- Higher DNA methylation within HIF3A measured in umbilical cord tissue was associated with greater infant birth weight and adiposity.

- The association of HIF3A methylation and birth weight remained significant after adjusting for cis-acting single nucleotide polymorphisms; but the association was stronger in one genotypic group.

- None of the prenatal environmental variables examined were consistently associated with HIF3A methylation and birth weight.

\section{Discussion}

- We have extended the previous finding that HIF3A DNA methylation in adults associates with BMI to show that the link between HIF3A DNA methylation with weight and adiposity can be detected at birth.

- Although pertaining to the same three CpGs within the HIF3A gene that were previously reported, our data were derived from a different tissue (umbilical cord vs blood and adipose), in a different population (Asian vs Caucasian), in a population-based cohort (normative range vs study population for metabolic disorders and controls) and most importantly at a different stage in the lifecourse (neonates vs adults).

- This suggests that prenatal factors may influence HIF3A methylation but despite the extensive data collected in the GUSTO, we were unable to definitively identify a responsible prenatal factor.

- We were unable to imply causality in our data and do not know whether adiposity causes HIF3A hypermethylation, HIF3A hypermethylation causes adiposity or if some independent factor influences both.

- In their study of HIF3A methylation and adult BMI, Dick et al. used Mendelian randomization to suggest that HIF3A hypermethylation is a consequence of increased adiposity. We could draw the same conclusion from our data but note that some of the assumptions of Mendelian randomization might not be satisfied.

- We suggest that as the association between HIF3A methylation and adiposity is detectable so early in life, HIF3A may be a potential biomarker of metabolic trajectory. 
M Hanson, CJ Henry, C-Y Hsu, H Inskip, J Kapur, K Kwek, IY-M Lau, BW Lee, N Lek, SB Lim, Y-L Low, I Magiati, LM Daniel, C Ngo, K Naiduvaje, WW Pang, A Qiu, BL Quah, VS Rajadurai, M Rauff, SA Rebello, JL Richmond, A RifkinGraboi, LP-C Shek, A Sheppard, B Shuter, L Singh, W Stunkel, $\mathrm{LL}$ Su, OH Teoh, HPS van Bever, RM van Dam, IBY Wong, PC Wong, GSH Yeo.

\section{Financial \& competing interests disclosure}

KM Godfrey, PD Gluckman and Y-S Chong have received reimbursement for speaking at conferences sponsored by companies selling nutritional products. They are part of an academic consortium that has received research funding from Abbott Nutrition, Nestec and Danone. This work was supported by the Translational Clinical Research (TCR) Flagship Program on Developmental Pathways to Metabolic Disease funded by the National Research Foundation (NRF) and administered by the National Medical Research Council (NMRC), Singapore - NMRC/TCR/004-NUS/2008. Additional funding is provided by the Singapore Institute for Clinical Sciences (SICS) - Agency for Science, Technology and Research (A*STAR), Singapore. KM Godfrey is supported by the National Institute for Health Research through the NIHR South-

\section{References}

Papers of special note have been highlighted as:

- of interest; $\bullet \bullet$ of considerable interest

1 Holbrook JD. An epigenetic escape route. Trends Genet. 31(1), 2-4 (2015).

- Review explaining the potential utility of epigenetic biomarkers for developmental trajectories.

2 Dick KJ, Nelson CP, Tsaprouni L et al. DNA methylation and body-mass index: a genome-wide analysis. Lancet 383(9933), 1990-1998 (2014).

-• Original epigenome-wide association study (EWAS) that linked HIF3A methylation to adult BMI.

3 Agha G, Houseman EA, Kelsey KT, Eaton CB, Buka SL, Loucks EB. Adiposity is associated with DNA methylation profile in adipose tissue. Int. J. Epidemiol. doi:10.1093/ije/ dyu236 (2014) (Epub ahead of print).

4 Hanson M, Godfrey KM, Lillycrop KA, Burdge GC, Gluckman PD. Developmental plasticity and developmental origins of noncommunicable disease: theoretical considerations and epigenetic mechanisms. Prog. Biophys. Mol. Biol. 106(1), 272-280 (2011).

5 Low FM, Gluckman PD, Hanson MA. Developmental plasticity and epigenetic mechanisms underpinning metabolic and cardiovascular diseases. Epigenomics 3(3), 279-294 (2011).

6 Gluckman PD, Hanson M, Zimmet P, Forrester T. Losing the war against obesity: the need for a developmental perspective. Sci. Transl. Med. 3(93), 93cm19 (2011).

7 Robinson SM, Crozier SR, Harvey NC et al. Modifiable early-life risk factors for childhood adiposity and overweight: an analysis of their combined impact and potential for prevention. Am. J. Clin. Nutr. 101(2), 368-375 (2015). ampton Biomedical Research Centre and by the European Union's Seventh Framework Programme (FP7/2007-2013), project EarlyNutrition under grant agreement no 289346. The authors have no other relevant affiliations or financial involvement with any organization or entity with a financial interest in or financial conflict with the subject matter or materials discussed in the manuscript apart from those disclosed.

No writing assistance was utilized in the production of this manuscript.

\section{Ethical conduct of research}

The authors state that they have obtained appropriate institutional review board approval or have followed the principles outlined in the Declaration of Helsinki for all human or animal experimental investigations. In addition, for investigations involving human subjects, informed consent has been obtained from the participants involved.

\section{Open access}

This work is licensed under the Attribution-NonCommercialNoDerivatives 4.0 Unported License. To view a copy of this license, visit http://creativecommons.org/licenses/by-nc-nd/4.0/

8 Gluckman PD, Hanson MA, Buklijas T, Low FM, Beedle AS. Epigenetic mechanisms that underpin metabolic and cardiovascular diseases. Nat. Rev. Endocrinol. 5(7), 401-408 (2009).

9 Heijmans BT, Tobi EW, Stein AD et al. Persistent epigenetic differences associated with prenatal exposure to famine in humans. Proc. Natl Acad. Sci. USA 105(44), 17046-17049 (2008).

10 Lillycrop KA, Slater-Jefferies JL, Hanson MA, Godfrey $\mathrm{KM}$, Jackson AA, Burdge GC. Induction of altered epigenetic regulation of the hepatic glucocorticoid receptor in the offspring of rats fed a protein-restricted diet during pregnancy suggests that reduced DNA methyltransferase-1 expression is involved in impaired DNA methylation and changes in histone modifications. Br. J. Nutr. 97(6), 1064-1073 (2007).

11 Boney CM, Verma A, Tucker R, Vohr BR. Metabolic syndrome in childhood: association with birth weight, maternal obesity, and gestational diabetes mellitus. Pediatrics 115(3), e290-e296 (2005).

12 Painter RC, Osmond C, Gluckman P, Hanson M, Phillips DI, Roseboom TJ. Transgenerational effects of prenatal exposure to the Dutch famine on neonatal adiposity and health in later life. BJOG 115(10), 1243-1249 (2008).

13 Ong ML, Lin X, Holbrook JD. Measuring epigenetics as the mediator of gene/environment interactions in dohad. J. Dev. Orig. Health Dis. 6(1), 10-16 (2015).

14 Teh AL, Pan H, Chen L et al. The effect of genotype and in utero environment on interindividual variation in neonate DNA methylomes. Genome Res. 24(7), 1064-1074 (2014). 
- Genome-wide survey of neonate methylomes showing influence of the interaction of genotypic and environmental influences. the GUSTO birth cohort study. Int. J. Epidemiol. 43(5), 1401-1409 (2012).

16 Bibikova M, Barnes B, Tsan C et al. High density DNA methylation array with single CPG site resolution. Genomics 98(4), 288-295 (2011).

17 Pan H, Chen L, Dogra S et al. Measuring the methylome in clinical samples: improved processing of the infinium human methylation 450 beadchip array. Epigenetics $7(10), 1173-1187$ (2012).

18 Dedeurwaerder S, Defrance M, Calonne E, Denis H, Sotiriou C, Fuks F. Evaluation of the infinium methylation $450 \mathrm{k}$ technology. Epigenomics 3(6), 771-784 (2011).

19 Johnson WE, Li C, Rabinovic A. Adjusting batch effects in microarray expression data using empirical Bayes methods. Biostatistics 8(1), 118-127 (2007).

20 Houseman EA, Accomando WP, Koestler DC et al. DNA methylation arrays as surrogate measures of cell mixture distribution. BMC Bioinformatics 13(1), 86 (2012).

- Reference method for adjusting associations of DNA methylation in tissues for cellular components.

21 Houseman EA, Molitor J, Marsit CJ. Reference-free cell mixture adjustments in analysis of DNA methylation data. Bioinformatics 30(10), 1431-1439 (2014).

- Reference-free method for adjusting associations of DNA methylation in tissues for cellular components and other unknown factors.

22 Leek JT, Johnson WE, Parker HS, Jaffe AE, Storey JD. The SVA package for removing batch effects and other unwanted variation in high-throughput experiments. Bioinformatics 28(6), 882-883 (2012).

- Surrogate variable method for adjusting associations of DNA methylation in tissues for cellular components and other unknown factors.

23 EBI.

www.ebi.ac.uk/ega/

24 Gutierrez-Arcelus M, Lappalainen T, Montgomery SB et al. Passive and active DNA methylation and the interplay with genetic variation in gene regulation. Elife 2, e00523 (2013).

25 Leek JT, Storey JD. Capturing heterogeneity in gene expression studies by surrogate variable analysis. PLoS Genet. 3(9), 1724-1735 (2007).

\section{Affiliations}

\section{- Hong Pan}

Singapore Institute for Clinical Sciences (SICS), A*STAR, Brenner

Centre for Molecular Medicine, 117609, Singapore

and

School of Computer Engineering, Nanyang Technological University

(NTU), 639798, Singapore
26 Leek JT, Scharpf RB, Bravo HC et al. Tackling the widespread and critical impact of batch effects in high-throughput data. Nat. Rev. Genet. 11(10), 733-739 (2010).

27 Harlid S, Xu Z, Panduri V, Sandler DP, Taylor JA. CPG sites associated with cigarette smoking: analysis of epigenome-wide data from the sister study. Environ. Health Perspect. 122(7), 673-678 (2014).

28 Barker M, Robinson S, Osmond C, Barker DJ. Birth weight and body fat distribution in adolescent girls. Arch. Dis. Child. 77(5), 381-383 (1997).

29 Persson M, Pasupathy D, Hanson U, Norman M. Birth size distribution in 3,705 infants born to mothers with Type 1 diabetes: a population-based study. Diabetes Care 34(5), 1145-1149 (2011).

30 Murphy TM, Mill J. Epigenetics in health and disease: heralding the EWAS era. Lancet 383(9933), 1952-1954 (2014).

-• Comment of Dick et al. [2], which elaborates on Mendelian randomization approach.

31 Chen L, Pan H, Tuan TA et al. Brain-derived neurotrophic factor $(B D N F)$ val66met polymorphism influences the association of the methylome with maternal anxiety and neonatal brain volumes. Dev. Psychopathol. 27(1), 137-150 (2015).

32 Gluckman PD, Hanson MA, Spencer HG, Bateson P. Environmental influences during development and their later consequences for health and disease: implications for the interpretation of empirical studies. Proc. Biol. Sci. 272(1564), 671-677 (2005).

33 Vickers MH. Developmental programming and transgenerational transmission of obesity. Ann. Nutr. Metab. 64(Suppl. 1), 26-34 (2014).

34 Barua S, Junaid MA. Lifestyle, pregnancy and epigenetic effects. Epigenomics 7(1), 85-102 (2015).

35 Pietilainen KH, Kaprio J, Rasanen M, Winter T, Rissanen A, Rose RJ. Tracking of body size from birth to late adolescence: contributions of birth length, birth weight, duration of gestation, parents' body size, and twinship. Am. J. Epidemiol. 154(1), 21-29 (2001).

36 Johnson L, Llewellyn CH, Van Jaarsveld CH, Cole TJ, Wardle $\mathrm{J}$. Genetic and environmental influences on infant growth: prospective analysis of the gemini twin birth cohort. PLoS ONE 6(5), e19918 (2011).

37 Demerath EW, Choh AC, Czerwinski SA et al. Genetic and environmental influences on infant weight and weight change: the Fels longitudinal study. Am. J. Hum. Biol. 19(5), 692-702 (2007).

\section{- Xinyi Lin}

Singapore Institute for Clinical Sciences (SICS), A*STAR, Brenner Centre for Molecular Medicine, 117609, Singapore

\section{- Yonghui Wu}

Singapore Institute for Clinical Sciences (SICS), A*STAR, Brenner Centre for Molecular Medicine, 117609, Singapore 


\section{- Li Chen}

Singapore Institute for Clinical Sciences (SICS), A*STAR, Brenner Centre for Molecular Medicine, 117609, Singapore

- Ai Ling Teh

Singapore Institute for Clinical Sciences (SICS), A*STAR, Brenner Centre for Molecular Medicine, 117609, Singapore

\section{- Shu E Soh}

Saw Swee Hock School of Public Health, National University of Singapore (NUS), 117597, Singapore

and

Yong Loo Lin School of Medicine, National University of Singapore (NUS), 119228, Singapore

- Yung Seng Lee

Singapore Institute for Clinical Sciences (SICS), A*STAR, Brenner Centre for Molecular Medicine, 117609, Singapore and

Yong Loo Lin School of Medicine, National University of Singapore (NUS), 119228, Singapore

and

Division of Paediatric Endocrinology \& Diabetes, Khoo Teck PuatNational University Children's Medical Institute, National University Health System, 119228, Singapore

- Mya Thway Tint

Yong Loo Lin School of Medicine, National University of Singapore (NUS), 119228, Singapore

- Julia L Maclsaac

Department of Medical Genetics, Centre for Molecular Medicine \& Therapeutics, Child \& Family Research Institute, University of British Columbia, Vancouver, BC, V5Z 4H4 Canada

\section{- Alexander M Morin}

Department of Medical Genetics, Centre for Molecular Medicine \& Therapeutics, Child \& Family Research Institute, University of British Columbia, Vancouver, BC, V5Z 4H4 Canada

\section{- Kok Hian Tan}

KK Women's and Children's Hospital, 229899, Singapore

- Fabian Yap

KK Women's and Children's Hospital, 229899, Singapore

- Seang Mei Saw

Saw Swee Hock School of Public Health, National University of Singapore (NUS), 117597, Singapore
- Michael S Kobor

Department of Medical Genetics, Centre for Molecular Medicine \& Therapeutics, Child \& Family Research Institute, University of British Columbia, Vancouver, BC, V5Z 4H4 Canada

\section{- Michael J Meaney}

Singapore Institute for Clinical Sciences (SICS), A*STAR, Brenner Centre for Molecular Medicine, 117609, Singapore

and

Ludmer Centre for Neuroinformatics \& Mental Health, Douglas University Mental Health Institute, McGill University, Montreal, (Quebec) H4H 1R3, Canada

\section{- Keith M Godfrey}

MRC Lifecourse Epidemiology Unit \& NIHR Southampton Biomedical Research Centre, University of Southampton \& University Hospital Southampton NHS Foundation Trust, Southampton, SO16 6YD, UK

\section{- Yap-Seng Chong}

Singapore Institute for Clinical Sciences (SICS), A*STAR, Brenner Centre for Molecular Medicine, 117609, Singapore and Yong Loo Lin School of Medicine, National University of Singapore (NUS), 119228, singapore

\section{- Peter D Gluckman}

Singapore Institute for Clinical Sciences (SICS), A*STAR, Brenner Centre for Molecular Medicine, 117609, Singapore and

Centre for Human Evolution, Adaptation \& Disease, Liggins Institute, University of Auckland, Auckland, 1142, New Zealand

\section{- Neerja Karnani}

Singapore Institute for Clinical Sciences (SICS), A*STAR, Brenner Centre for Molecular Medicine, 117609, Singapore and

Yong Loo Lin School of Medicine, National University of Singapore (NUS), 119228, Singapore

\section{- Joanna D Holbrook}

Singapore Institute for Clinical Sciences (SICS), A*STAR, Brenner Centre for Molecular Medicine, 117609, Singapore

- GUSTO Study Group 